\title{
Vortex-induced Vibration of a Flexible Free-hanging Circular Cantilever
}

\author{
R. W. Prastianto ${ }^{1}$, K. Otsuka ${ }^{2} \&$ Y. Ikeda ${ }^{2}$ \\ ${ }^{1}$ Department of Ocean Engineering, Institut Teknologi Sepuluh Nopember (ITS), \\ Surabaya, Indonesia \\ ${ }^{2}$ Department of Marine System Engineering, Osaka Prefecture University, Japan
}

\begin{abstract}
The behavior of a free-hanging riser of floating offshore structures would be different from a typical at-sea-floor-terminated riser type of oil or gas platforms. For the design purpose, the present study was intended to incorporate some important factors of the riser conditions (i.e. bidirectional vibration, freeend condition, and spanwise variation of response amplitude) for investigating its dynamics characteristics. An experimental investigation on time-dependent motion of a flexible free-hanging circular cantilever subjected to uniform crossflows has been carried out. The free-end condition cantilever has a 34.4 aspect ratio and a low mass ratio of about 1.24. The cylinder freely oscillates in both inline and transverse to the flow. Reynolds number varied from 10,800 to 37,800 . The "jump phenomenon" was found in the inline motion of the cylinder that agrees well with an existing comparable work. At high flow velocities, the 3rd higher harmonic frequencies of the cylinder transverse response became predominant that produce quite different motion characteristics compared to the other existing comparable works with 2-dimensional bottom-end condition. Generally, the results suggested that the flexible free-hanging cantilever generate different vortex wake mode than either, a uniform (a short-rigid flexiblymounted cylinder) or a linear amplitude variation along the span case (a pivoted cylinder).
\end{abstract}

Keywords: vortex-induced vibration; free-hanging cantilever; bidirectional motion; free-end condition; vortex wake mode.

\section{Introduction}

So far, general characteristics of a flexible circular cylinder subjected to water cross-flows are not well understood in various parameters. Many factors strongly influenced the cylinder dynamics, such as Reynolds number range, aspect ratio, mass ratio, and the motion degrees of freedom of the cylinder, etc. A large number of studies are still required before one can quantify the governing parameters for wider practical applications.

During the early studies on the oscillating cylinder, a short-rigid flexiblymounted cylinder was commonly used in forced or free vibration tests, and typically the cylinder motion allowed in only one direction, while restricted in

Received February $9^{\text {th }}, 2009$, Revised May $11^{\text {th }}, 2009$, Accepted for publication August $2^{\text {nd }}, 2009$. 
another [1-4]. More specific, the effects of mass and damping on the cylinder dynamics were then also investigated [5-6]. Still using a short-rigid cylinder, some extension works in which the cylinder is allowed to oscillate in-line and transverse simultaneously to the fluid flow were introduced. Some significant differences on the cylinder dynamics characteristics from either the stationary or 1-direction only motion cylinder case were found [7-11]. For stationary and flexible cylinders, it was proved that the effect of the cylinder-end boundary is very important on the characteristics of the fluid flow, then in turn on measured drag and lift forces, and the cylinder response [3, 12]. More recently, some experimental studies on a low mass ratio freely vibrating cylinder were performed, but the conditions were only for 1-directional motion and within relatively low Reynolds numbers $(1,500<R e<21,000)$. Additionally, the bottom-end of the cylinder was conditioned by a tiny gap to the tank floor, in order to obtain a 2-dimensional effect of the flow [13-15].

Several experimental works on the vortex-induced vibration of a flexible cantilever cylinder with free-end condition in water flows were initiated many years ago. However, in some of those works [16, 17], due to limited experimental conditions, the orbits of the cylinder response and induced hydrodynamic forces could not be obtained.

In the case of floating offshore structures having a free-hanging type of riser, such as ocean thermal energy conversion (OTEC) or carbon dioxide $\left(\mathrm{CO}_{2}\right)$ sequestration platforms, the riser behavior would be different from a typical atsea-floor-terminated riser type of oil or gas platforms. For the design purpose, a better understanding on the dynamics of such kind of a flexible free-hanging riser is definitely required. This present experimental work, therefore, is intended to incorporate some important factors that relevant to these structures which are the bidirectional vibration, free-end condition, and spanwise variation of amplitude of the cylinder. Results of this study are expected can be utilized as basic considerations in the preliminary design stage of such structures.

This paper presents new results on the characteristics of time-dependent motion of a flexible free-hanging circular cantilever. Meanwhile, the induced hydrodynamic forces acting on the same test cylinder, but no accelerometers installed, have been investigated in [18].

\section{Experimental Conditions}

The experiment was carried out in a towing tank of the Department of Marine System Engineering at Osaka Prefecture University, Japan. The tank was 70.0 $\mathrm{m}$ long with an effective running distance of about $45 \mathrm{~m}, 3.0 \mathrm{~m}$ wide and $1.5 \mathrm{~m}$ 
deep; it was equipped by a main towing carriage with a maximum towing speed of $2.5 \mathrm{~m} / \mathrm{sec}$.

The test cylinder was made of a standard polyvinyl chlorite (PVC) pipe. The material and section properties of the cylinder are shown in Table 1. The cylinder has a total aspect ratio (total length-to-diameter ratio, $\mathrm{AR}_{\mathrm{tot}}$ ) of 34.4, and a mass ratio (the ratio of cylinder mass to displaced water mass, $m^{*}$ ) of about 1.24 .

Table 1 Properties of the test cylinder.

\begin{tabular}{lc}
\hline \multicolumn{1}{c}{ Items } & Properties \\
\hline Total length, $L(\mathrm{~m})$ & 1.65 \\
Wet length, $L_{\text {wet }}(\mathrm{m})$ & 1.27 \\
Outside diameter, $D(\mathrm{~mm})$ & 48.0 \\
Wall thickness, $t_{\mathrm{w}}(\mathrm{mm})$ & 4.0 \\
Mass per unit length in air $(\mathrm{kg} / \mathrm{m})$ & 0.764 \\
\hline
\end{tabular}

The top-end of the cylinder was connected to a 2-component load cell which is fixed to adjustable beams mounted on the towing carriage (Figure 1(a)). The load cells were used for measuring the hydrodynamic forces acting on the test cylinder in both, in-line (drag force) and transverse (lift force) directions simultaneously. Meanwhile, to measure the inline and transverse accelerations of the cylinder, 2 small dimensions of 1-direction waterproof accelerometers (Kyowa ASW-A type) were mounted inside the bottom-end of the cylinder. Each accelerometer has approximately 40 grams of weight excluded the cable, $18 \times 18 \times 24 \mathrm{~mm}$ in dimensions, and has up to $490.3 \mathrm{kPa}$ of water pressure resistance. As an initial condition, the cylinder was partially submerged $\left(L_{\mathrm{wet}}=\right.$ $1.27 \mathrm{~m}$, to give a wet aspect ratio, $\mathrm{AR}_{\text {wet, }}$ of 26.5) into the still water from the towing carriage as a hanging vertical cantilever with $0.23 \mathrm{~m}$ gap (allowing 3dimensional effect of the flow) from the bottom of the tank. Figure 1 illustrates the experimental setup and nomenclature of the flow-cylinder system.

To generate uniform cross-flows, the carriage was moved along the tank with the test cylinder fixed to the carriage. The ratio of the cylinder diameter to tank width was $16: 1000$, so that some disturbances which come into the system due to the wall (e.g. reflection wave, etc) are negligible. The towing speeds $(U)$ were changed from 0.20 up to $0.70 \mathrm{~m} / \mathrm{sec}$ by an increment of 0.05 that approximately correspond to the Reynolds number $(R e)$ of $10,800 \sim 37,800$. The Reynolds number is defined as $R e=U D / v$, where $v$ is the kinematics viscosity of the water. The kinematics viscosity is assumed to be $0.89 \times 10^{-6} \mathrm{~m}^{2} / \mathrm{sec}$ for this experiment. During the towing for each case of the test, the cylinder oscillated in 2 degrees of freedom as a self-excited motion system. 
The experimental data were acquired through a standard data acquisition system with sampling rate of $50 \mathrm{~Hz}$ and low-pass filtered of $20 \mathrm{~Hz}$. Four channels were used to record the forces and response data of the cylinder. The raw signals were digitally low-pass filtered by a cut-off frequency of $6 \mathrm{~Hz}$ in order to isolate the low modes. The data used for the calculation of the intended parameters were based on a selected range from approximately stationary data within the whole time during the towing for each case. The Fast Fourier Transform (FFT) procedure was applied to analyze frequency content of the measured time series data. For the motion of the cylinder, prior to integrating the accelerations time series data into the displacement time history, the accelerations data were lowpass filtered in order to remove their high frequency noise. The normalized displacement amplitude (divided by $D$ ) were computed from their root mean square $(r m s)$ values as $A_{x}=\sqrt{ } 2 \times(x / D)_{r m s}$ and $A_{y}=\sqrt{ } 2 \times(y / D)_{r m s}$ for inline and cross-flow directions, respectively [15]. Finally, some analyzed parameters are presented as a function of the reduced velocity which is defined as $U r=$ $U / f_{\text {n.water }} D$, where $f_{\text {n.water }}$ is the fundamental (1st mode) natural frequency of the cylinder in still water.
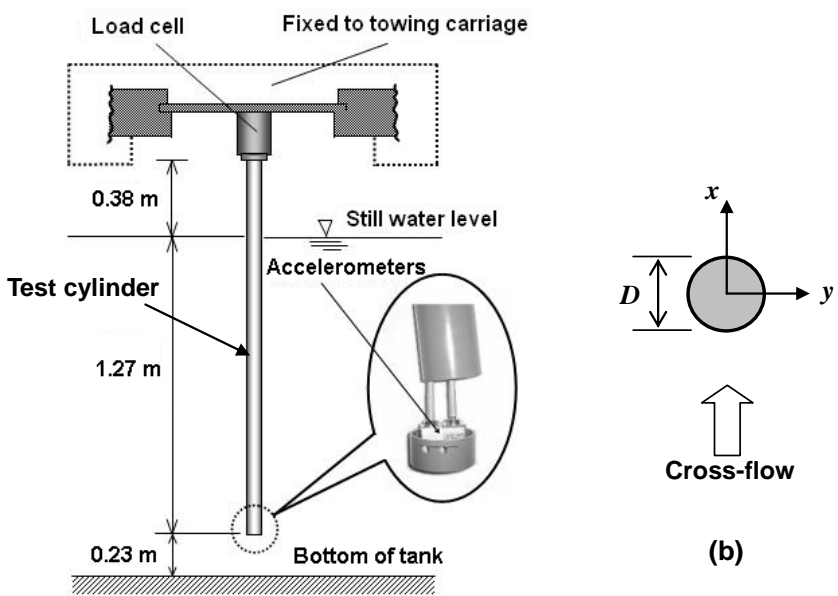

(a)

Figure 1 (a) Schematic view of experimental setup with close-up view of the accelerometers installed inside the bottom-end of the cylinder. (b) Nomenclature of the test cylinder immersed in uniform cross-flows.

Several free decay tests were performed in order to measure the fundamental natural frequencies of the test cylinder. To ensure the consistency of the results, the tests were performed 4 times in air and 3 times in water. In addition, some of the tests in air condition were carried out in both $x$ and $y$ directions, to ensure a uniformity of the natural frequencies in those two directions. The tests 
produced a unidirectional mean value of the fundamental natural frequency of $4.20 \mathrm{~Hz}$ and $2.15 \mathrm{~Hz}$ for in air and water condition, respectively. There was approximately $50 \%$ decrease on the cylinder's natural frequency for the still water case.

\section{Experimental Results and Discussion}

\subsection{Motion Amplitudes}

Motion amplitudes (normalized by $D$ ) at the bottom-end of the test cylinder in inline and transverse directions to the flow are displayed in Figure 2. At low $U r$ $(U r<3.39)$, the inline amplitudes $\left(A_{x}\right)$ are relatively larger than the transverse amplitudes $\left(A_{y}\right)$; the responses are dominated by the inline motion while the transverse motions relatively have not been developed yet, as could be confirmed later by their motion trajectories in Figure 7. The amplitude of the vibration in those two directions reaches nearly equal values at $3.39 \leq U r \leq$ 4.36. At higher regime of $U r(U r>4.36)$, the inline amplitudes, again, gradually become larger and larger than the transverse one, even though the transverse motions have also been stimulated and increase. Inline-transverse amplitudes ratios become larger as the $U r$ increases. For instance, at highest $U r(=6.79)$, the ratio reaches approximately 1.4. One reason is due to the mean drag force driving the cylinder to displace larger as the $U r$ increases. Basically, the scatter data of the motion amplitudes are quite fit to the quadratic polynomial functions as can be seen in Figure 2.

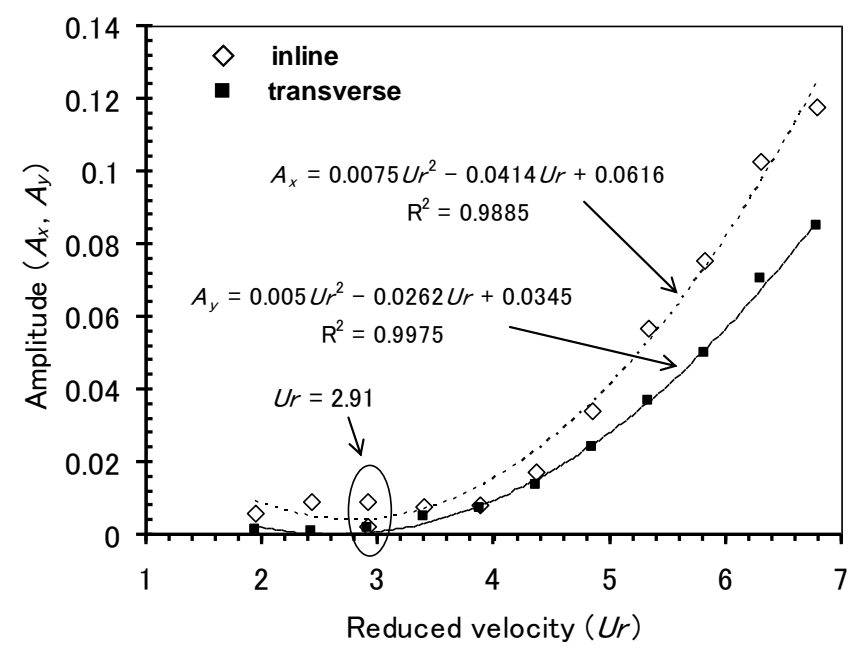

Figure 2 Normalized motion amplitudes at the bottom-end of the test cantilever in inline and transverse directions to the flows. 
However, at $U r=2.91$, there is a peculiar phenomenon in the inline motion that does not occur within the higher $U r$. There are two significant distinct amplitudes (double amplitude response) within the whole time history of the recorded response in the inline direction to the flow (in turn occurs in the corresponding force signal) as displayed in Figure 3. Within approximately first $35 \mathrm{sec}$, the amplitude of the response is smaller, and then followed by larger amplitude for remaining time of the response. The larger amplitude is approximately 4.5 times the smaller one (see also Figure 2).
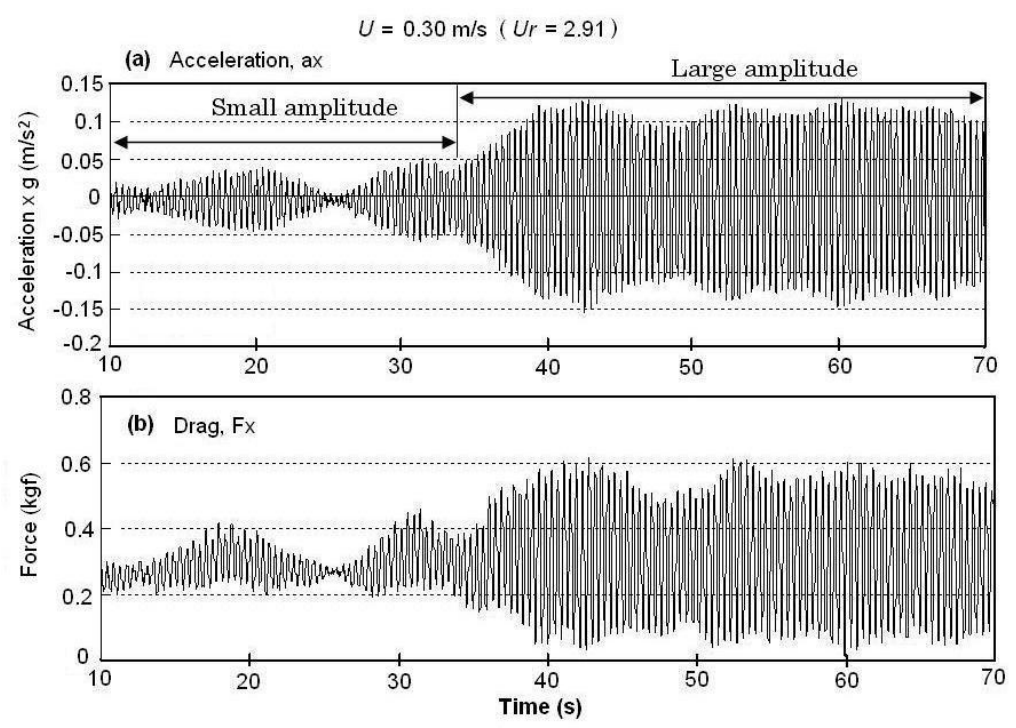

Figure 3 Time series of the motion acceleration (a), and the corresponding drag force (b), in inline direction describing the "jump phenomenon" on their amplitudes at the $U r=2.91 \mathrm{~g}$ is the gravitational acceleration $\left(\approx 9.81 \mathrm{~m} / \mathrm{s}^{2}\right)$.

This phenomenon agrees well with that was observed by Pesce and Fujarra [16] as the "jump phenomenon" of the cylinder motion. Their experiment used a similar flexible free-hanging cantilever with free-end condition, but the aspect ratio (wet aspect ratio, $\mathrm{AR}_{\text {wet }}$ ) and the mass ratio $\left(m^{*}\right)$ are about 95 and 2.36, respectively. These values are larger than the present model by approximately $70 \%$ and $50 \%$, respectively. They found the phenomenon from their time history signals of the strain, at two successive Ur of 8.336 and 8.380. However, it can be noted that the phenomenon found in the present work is somewhat different compared to the result of the work of that Pesce and Fujarra [16]. Here, the "jump phenomenon" occurs at a lower Ur and the response within the small amplitude portion shows more beating vibration rather than the stationary response. The aspect ratio and mass ratio of the cylinder can be expected as the factors that responsible to such difference. 
In relation to the low-mass ratio of bidirectional vibrating flexibly-mounted cylinder, Jauvtis and Williamson [9] found a dramatic change in fluid-cylinder interactions when mass ratios are reduced below a factor of 6 . Figure 4 shows some results from Jauvtis and Williamson [9] and Flemming and Williamson [21] for two different cylinder conditions. Figures 4(a) and 4(b) are for a uniform response amplitude (a short-rigid flexibly-mounted cylinder), and Figure 4(c) is for a case of linear amplitude variation along the span (a pivoted cylinder).

For a mass ratio of 2.6 (Figure 4(a)), a new response branch (as "super-upper" branch, symbolized by "SU") appears in transverse direction with massive amplitudes of about 1.5 diameters. This response corresponds with a new periodic vortex wake mode, "2T mode", which comprises a triplet of vortices being formed in each half cycle of vibration (Figure 4(b)).

(a)

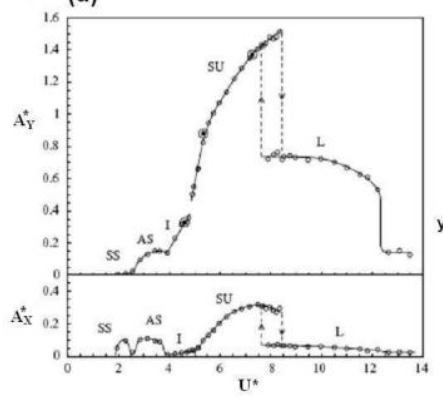

(b)

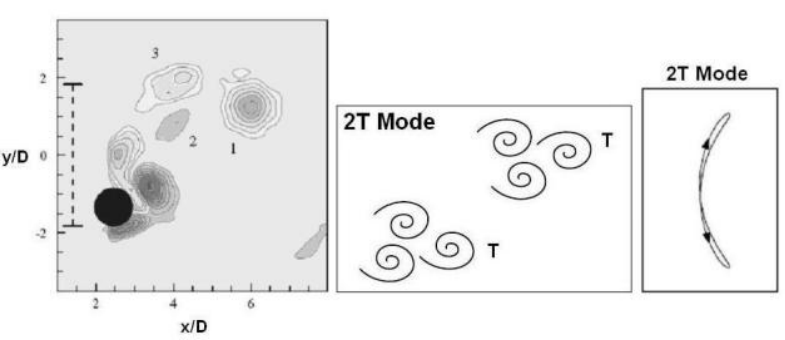

(c) 2C Mode

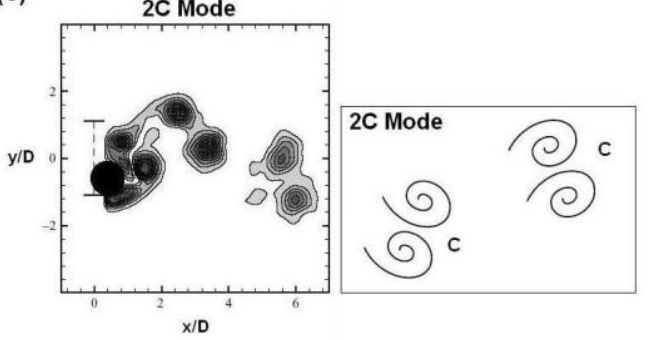

Figure 4 (a) A "super-upper" branch (SU) of high-amplitude response that appears for bidirectional cylinder vibration, $m^{*}=2.6$. (b) A corresponding a vortex wake mode "2T". Vortices no. 1 and 2 turn clockwise, while vortex no. 2 turns anticlockwise [9]. (c) A distinct new mode comprising two co-rotating vortices formed each half cycle (" $2 \mathrm{C}$ " mode) observed for a pivoted cylinder, plastic cylinder, $m^{*}=1.03$ [21]. The vortices are recorded by digital particle image velocimetry (DPIV) technique. 
If we compare Figure 2 and Figure 4(a), some differences can be found. For the present case, with much lower mass ratio of about 1.24 (about 50\% smaller), at high $U r$, the larger response is found in the inline response, in contrast to the case displayed in the Figure 4(a). Also, at the same $U r \approx 7$, the inline amplitude is only approximately 10 times smaller than that of the transverse amplitude for the flexibly-mounted cylinder case (Figure 4(a)). If we take a look on the trend of the response, perhaps the $U r$ range in the present test only covers the initial branch from the whole response of the present case, including the lower branch (following the terminology introduced in Figure 4(a) by a symbol of "I" and "L" for Initial and Lower branch, respectively). The SU branch could be reached after the $U r$ around 9 or more, because in the present much lower mass ratio case, the response peak either in inline or transverse direction as a function of the $U r$ is late to be reached.

\subsection{Motion Frequencies}

The motion frequency of the cylinder can be used to understand its motion trajectory. Figures 5 and 6 depict the power spectral density (PSD) of the motion accelerations in inline $(a x)$ and transverse (ay) directions as a function of $U r$. For all cases, the dominant frequencies clearly appear as narrow-banded frequency.

In streamwise direction, the response appear as single predominant frequencies for all values of $U r$ and the frequency linearly increases with the $U r$ as shown in the left column of Figures 5 and 6. Meanwhile, the transverse responses have various dominant frequencies depending on the $U r$. For first two $U r, 1.94$ and 2.42 , only a single predominant frequencies having same magnitude appears, as well as their frequencies in the streamwise direction, but with relatively smaller amplitudes.

Regarding its frequency, the case at $U r=2.91$, again, becomes a special one. Beside the "jump phenomenon" appears in the inline response, its response in transverse direction is also unique. Frequency content of the transverse response consists of 3 dominant frequencies in which the first frequency is the predominant one (Figure 5(f)). The first frequency typically corresponds to vortex shedding (or Strouhal) frequency with a value of half of the corresponding inline response frequency. The second frequency is approximately twice the first frequency, and corresponds to the inline response frequency itself. Meanwhile, the third frequency is a third higher harmonic frequency which has magnitude of approximately tripled the Strouhal one. 

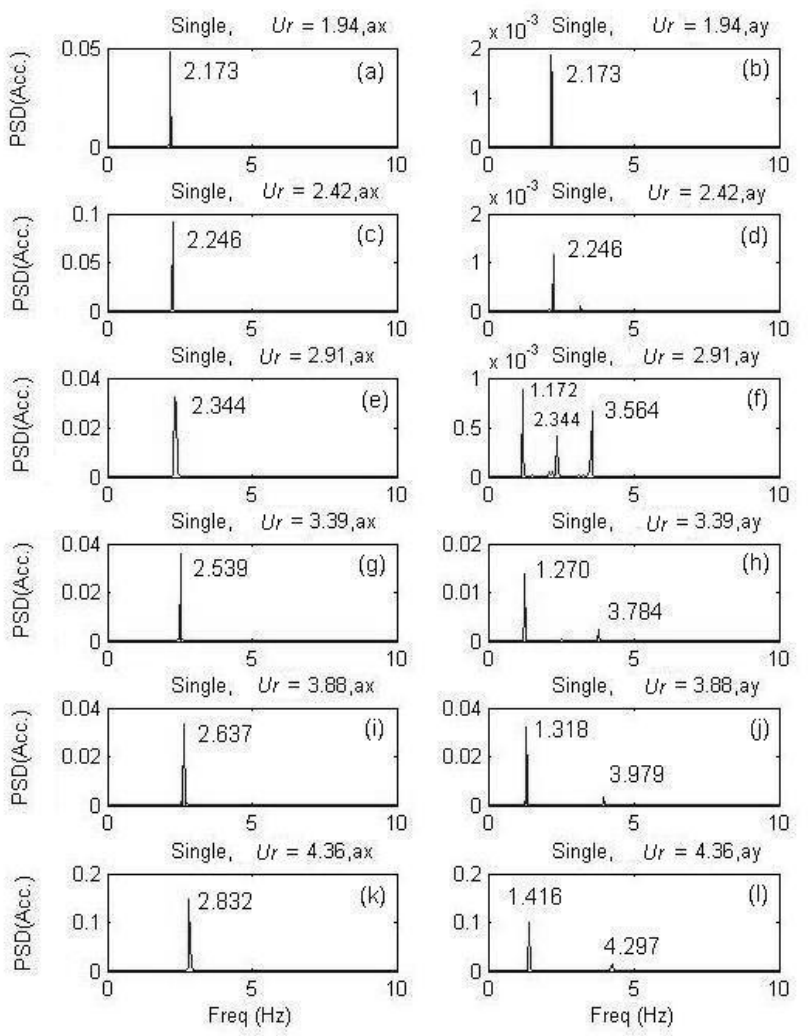

Figure 5 Power spectral density (PSD) of the motion accelerations at the bottom-end of the cylinder in inline $(a x)$ and transverse $(a y)$ directions for $U r=$ 1.94 to 4.36 .

As the $U r$ increases in each case, at $3.39 \leq U r \leq 6.79$, frequency content of the transverse response changes, in which the second frequency completely disappears and the remaining frequencies are only the first and third higher harmonic, respectively. Beside the first frequencies step by step linearly increase with the $U r$; the predominant frequencies can changeover at a certain $U r$, where the third harmonic frequencies become predominant (Figures 5 and 6). The third higher harmonic frequencies become the predominant one at $U r \geq$ 5.33 (Figure 6(d)). These different frequency conditions are obviously responsible for the trajectories change of the cylinder motion. The origin of this third higher harmonic excitation possibly can be addressed to the existence of a different vortex shedding mode in the present case. 

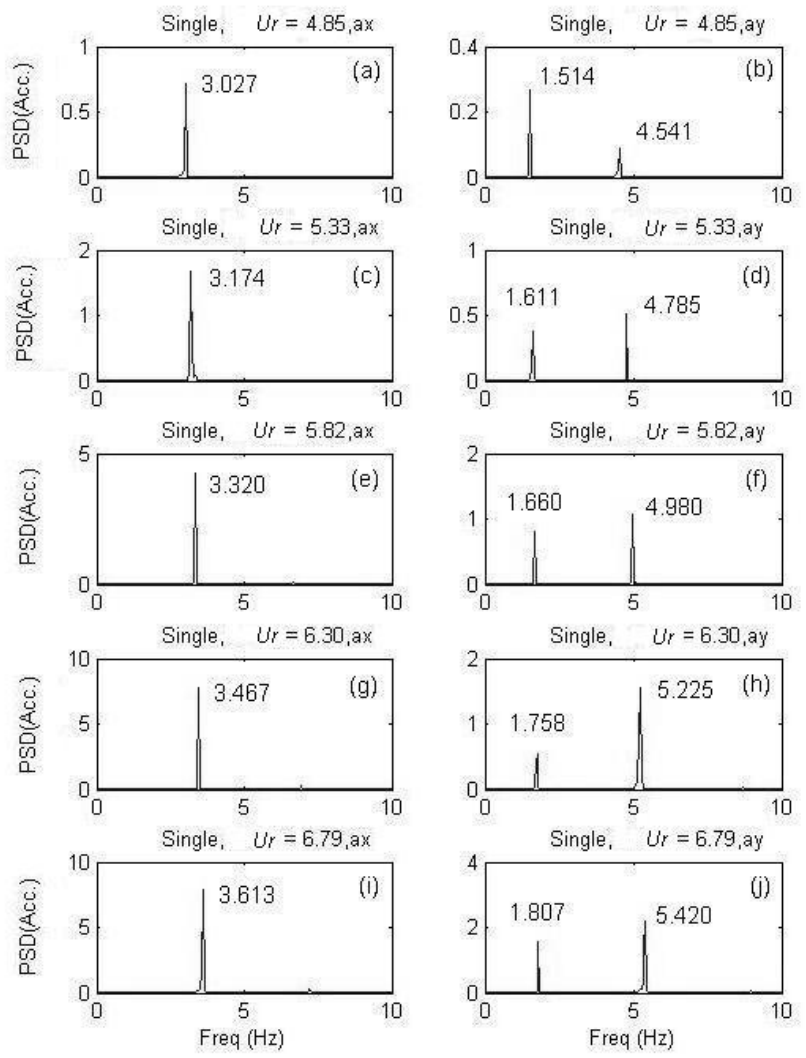

Figure 6 Power spectral density (PSD) of the motion accelerations at the bottom-end of the cylinder in inline $(a x)$ and transverse (ay) directions for $U r=$ 4.85 to 6.79 .

\subsection{Motion Trajectories}

The displacements in two directions, $\mathrm{x}(\mathrm{t}) / D$ and $\mathrm{y}(\mathrm{t}) / D$, can be plotted in 2-D plane to observe trajectories change of the cylinder motion, as well as their displacement magnitudes. Figure 7 shows the trajectories at the bottom-end of the cylinder. Each inset figure represents the trajectory pattern for a certain $U r$ value.

Basically, the trajectory patterns of the motion displacements clearly change with the parameter $U r$. As previously described (see Figure 2), at $1.94 \leq U r \leq$ 2.91, the motions are almost as inline-dominated response (Figure 7). At $U r=$ 3.39, the transverse response gradually increases and new different trajectory patterns are then developed, gradually approaching "a right-bended figure-of- 
eight" patterns at $U r=3.88$ and 4.36 , that nearly similar to the orbit created by a "2T" vortex mode (see Figure 4(b)).

Furthermore, in the range $4.85 \leq U r \leq 6.79$, other new different motion trajectories are created (Figures $7(\mathrm{~g})$ to $(\mathrm{k})$ ). New three intersection points within the trajectories, two points located symmetrically to the zero horizontal axes and another point at the "tail" of the trajectories, appear for $4.85 \leq U r \leq$ 5.82. As the $U r$ increases, in the range of $6.30 \leq U r \leq 6.79$, one more intersection point is created at the "tail" part of the trajectories. Total of five intersection points appear in this range of $U r$. In addition, such those trajectories much more no longer fit to typical orbit for VIV problems as e.g., the "perfect figure-of-eight". These evidences enrich the variability of the motion behavior of a low-mass flexible cylinder in water cross-flows, especially in the case of free-end condition with two-degree-of-freedom motion. However, at a certain $U r(U r=3.88)$, the trajectory pattern relatively similar to other case with higher $\operatorname{Re}$ of 103,000 at $U r=4.1$, as found, for instance, in the work of Lee et al. [19]. Such those varieties mean that parameter $U r$ strongly affects both the trajectory pattern and magnitude change of the cylinder motion.
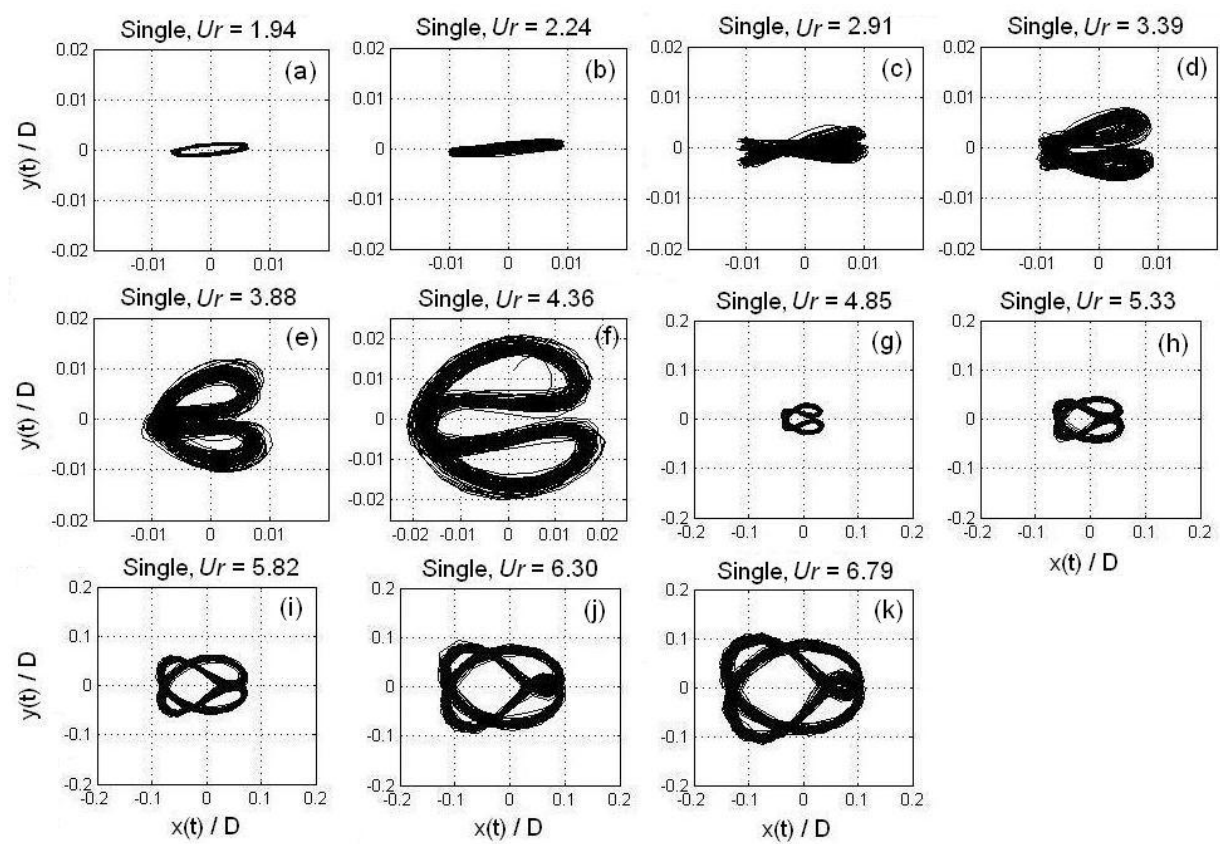

Figure 7 Motion trajectories at the bottom-end of the test cantilever as a function of the reduced velocity $(U r)$; Flow is left to right. 
Regarding the motion orbit of the flexible cylinder in general, many researchers reported various motion behaviors of a vibrating cylinder, even due to uniform flows. With a single cylinder towed horizontally in a towing tank with corresponding $R e$ of approximately 18,000, Wilde and Huijsmans [20] found a "lying banana" type of motion trajectory at the pipe mid rather than general pattern of "figures-of-eight" or "standing bananas". For a two-end supported vertical cylinder tested in a circulating current thank with high $R e$ of 76,000 to 265,000, Lee et al. [19] observed that the motion trajectories at a midpoint of the bare cylinder step by step change from predominantly inline motion, to figure-of-eight motion, to predominantly cross-flow motion as the $R e$ increases. They concluded that the displacement patterns are associated with the elastic mode shape and natural frequencies of the cylinder. Meanwhile, for a flexiblemounted rigid cylinder tested in flow channel which is allowed to have both inline and transverse motions, at $U r=8.64$, the trajectory plot of distinct pattern called "a narrow wind-blown figure-of-eight" was created with motion amplitude of above $0.7 D[10]$.

In the present experiment, besides the aspects of aspect ratio and mass ratio, another aspect, e.g. a spanwise variation of amplitude can also be expected influences the vortex wake mode, in turn the motion patterns, if compared to, such as the work of Jauvtis and Williamson [9], particularly for higher $U r$. As can be seen in the right side of Figure 4(b), the motion trajectory created by a " $2 \mathrm{~T}$ " vortex mode is a typical slightly right-bended "figure-of-eight" that completely different with the motion orbits of the present cantilever case for $U r$ $\geq 4.85$, although both of these works have the same bidirectional type of motion. This evidence suggests that the present test condition could be able to produce a different vortex mode.

Another work can support this analysis. Using a pivoted cylinder freely moves inline and transverse to the flow, Flemming and Williamson [21] observed different modes along the span. For the lightest (plastic cylinder with $m^{*}=$ 1.03) of their cylinders, they found a distinct new mode comprising two corotating vortices formed each half cycle, namely "2C mode" (Figure 4(c)). Nevertheless, we can also note some different conditions of the test compared to the present case, which are 2-D flow around the bottom-end of the cylinder, lower Reynolds number $(R e \approx 1000)$, and a linear amplitude variation along the span that in contrast to the flexible cantilevered cylinder having a nonlinear amplitude variation along the span. Apparently, due to such different factors involved in the system, we can suspect that a flexible free-hanging cantilever in present test will generate different vortex wake mode than either " $2 \mathrm{~T}$ " or " $2 \mathrm{C}$ " mode, at least based on the observed results. 
Finally, such findings give us insights that various phenomena involved in the problem. Many parameters and specific conditions affect the motion behavior of the flexible free-hanging cantilever subjected to uniform water flows. Further investigations are needed for better understanding of the problem. One possible effort to clarify the precise mechanism behind the observed phenomena is through a visualization study on the vortex wake modes occurred along the length of the cylinder, including at the wake around the cylinder's bottom-end.

\section{Conclusions}

The conclusions of the present work can be summarized as follows:

1. The cylinder response was strongly dictated by the reduced velocity parameter, Ur. The double amplitudes phenomenon, the "jump phenomenon", was found in the inline motion that agrees well with an existing comparable work. However, due to distinct conditions in the test, the phenomenon occurred at lower $U r$ than the existing work.

2. At high $U r(U r \geq 5.33)$, the third higher harmonic frequencies of the transverse response became predominant. This condition obviously could be expected due to the change on the motion trajectory patterns of the cylinder motion.

3. Free bottom-end condition, higher Reynolds number, and a nonlinearity of amplitude variation along the span (due to the cantilevered condition of the cylinder) involved in the present experiment, produced quite different motion characteristics compared to other existing comparable works. It seems that the present test generate different vortex wake mode than either, " $2 \mathrm{~T}$ " mode (in the case of a short-rigid flexibly-mounted cylinder) or " $2 \mathrm{C}$ " mode (in the case of a rigid pivoted cylinder). A visualization study on the vortex wake modes need to be performed, to clarify the precise mechanism behind the observed phenomena.

\section{Acknowledgements}

The first author wishes to thank Japan's Ministry of Education, Culture, Sports, Science and Technology for providing a scholarship for his doctoral study at the Department of Marine System Engineering, Osaka Prefecture University, Japan. The permissions from Indonesia's Ministry of National Education and Institut Teknologi Sepuluh Nopember (ITS), Surabaya are sincerely appreciated.

\section{References}

[1] Bearman, P. W. \& Obasaju, E. D., Transverse forces on a circular cylinder oscillating in-line with a steady current, Proc. 8th International 
Conference on Offshore Mechanics and Arctic Engineering, The Hague, pp. 253-258, 1989.

[2] Moe, G. \& Wu, J., The lift force on a cylinder vibrating in a current, Journal of Offshore Mechanics and Arctic Engineering (JOMAE), 112, 297-303, 1990.

[3] Khalak, A. \& Williamson, C. H. K., Dynamics of a Hydroelastic Cylinder with Very Low Mass and Damping, Journal of Fluids and Structures, 10, 455-472, 1996.

[4] Khalak, A. \& Williamson, C. H. K., Fluid Forces and Dynamics of a Hydroelastic Structure with Very Low Mass and Damping, Journal of Fluids and Structures, 11, 973-982, 1997a.

[5] Khalak, A. \& Williamson, C. H. K., Investigation of relative effects of mass and damping in vortex-induced vibration of a circular cylinder, Journal of Wind Eng. and Industrial Aerodynamics, 69-71, 341-350, 1997b.

[6] Khalak, A. \& Williamson, C. H. K., Motion, Forces and Mode Transitions Structure in Vortex-induced Vibrations at Low MassDamping, Journal of Fluids and Structures, 13, 813-851, 1999.

[7] Sarpkaya, T., Hydrodynamic Damping, Flow-induced Oscillation, and Biharmonic Response, Journal of Offshore Mechanics and Arctic Engineering (JOMAE), 117, 232-238, 1995.

[8] Jeon, D. \& Gharib, M., On circular cylinders undergoing two-degree-offreedom forced motions, Journal of Fluids and Structures, 15, 533-541, 2001.

[9] Jauvtis, N., \& Williamson, C. H. K., The effect of two degrees of freedom on vortex-induced vibration at low mass and damping, Journal of Fluids Mechanic, 509, 23-62, 2004.

[10] Blevin, R. D. \& Coughran, C. S., Experimental Investigation of VortexInduced Vibration in Two-Dimensions, Proc. 18th International Offshore and Polar Engineering Conference (ISOPE2008), Vancouver, Canada, pp. 475-480, 2008.

[11] Sanchis, A., Salevik, J. \& Grue, J., Two-degree-of-freedom vortexinduced vibrations of a spring-mounted rigid cylinder with low mass ratio, Journal of Fluids and Structures, 24, 907-919, 2008.

[12] Kitagawa, T., Fujino, Y. \& Kimura, K., Effects of Free-end Condition on End-cell-induced Vibration, Journal of Fluids and Structures, 13, 499518, 1999.

[13] Branković, M. \& Bearman, P. W., Measurements of transverse forces on circular cylinders undergoing vortex-induced vibration, Journal of Fluids and Structures, 22, 829-836, 2006.

[14] Assi, G. R. S., et al., Experimental investigation of flow-induced vibration interference between two circular cylinders, Journal of Fluids and Structures, 22, 819-827, 2006. 
[15] Assi, G. R. S., et al., Unsteady force measurements on a responding circular cylinder in the wake of an upstream cylinder, Proc. 26th International Conference on Offshore Mechanics and Arctic Engineering, San Diego, USA, Paper no. OMAE2007-29040, 2007.

[16] Pesce, C. P. \& Fujarra, A. L. C., Vortex-induced Vibration and Jump Phenomenon: Experiments with a Clamped Flexible Cantilever in Water, International Journal of Offshore and Polar Eng., 10(1), 26-33, 2000.

[17] Fujarra, A. L. C., et al., Vortex-induced Vibration of a Flexible Cantilever, Journal of Fluids and Structures, 15, 651-658, 2001.

[18] Prastianto, R. W, Otsuka, K. \& Ikeda, Y., Effects of Free-end Condition on Hydrodynamic Forces of a Flexible Hanging-off Circular Cylinder Undergoing Vortex-induced Vibration, Proc. JASNAOE Annual Conference, Nagasaki, Japan, pp. 259-262, 2008.

[19] Lee, L., Allen, D. W. \& Henning, D. L. Y., Motion Trajectory of Bare and Suppressed Tubulars Subjected to Vortex Shedding at High Reynolds Numbers, Proc. 14th International Offshore and Polar Engineering Conference (ISOPE2004), Toulon, France, pp. 517-523, 2004.

[20] Wilde, J. J. D. \& Huijsmans, R. H. M., Laboratory Investigation of Long Riser VIV Response, Proc. 14th International Offshore and Polar Engineering Conference (ISOPE2004), Toulon, France, pp. 511-516, 2004.

[21] Flemming, F. \& Williamson, C. H. K., Vortex-induced vibration of a pivoted cylinder, Journal of Fluid Mechanics, 522, 215-252, 2005. 